\begin{tabular}{lll} 
Universidade do Minho & Universidade do Minho \\
Escola de Ciências & $\begin{array}{l}\text { Universidade do Minho } \\
\text { Escola de Ciências }\end{array}$ \\
Centro de Matemática & Departamento de Matemática e Aplicações \\
\hline
\end{tabular}

\title{
Polynomials over Quaternions and Coquaternions: A Unified Approach
}

\author{
M. Irene Falcão ${ }^{a, c} \quad$ Fernando Miranda $^{a, c} \quad$ Ricardo Severino $^{c} \quad$ M. Joana Soares ${ }^{b, c}$ \\ ${ }^{a}$ CMAT - Centre of Mathematics, University of Minho, Portugal \\ ${ }^{b}$ NIPE - Economic Policies Research Unit, University of Minho, Portugal \\ ${ }^{c}$ Department of Mathematics and Applications, University of Minho, Portugal
}

\section{Information}

\section{Keywords:}

Quaternions; coquaternions; polynomials; zeros.

Original publication:

Lecture Notes in Computer Science, vol. 10405, pp. 379-393, 2017

DOI: $10.1007 / 978-3-319-62395-5 \_26$

www.springerlink.com

\begin{abstract}
This paper aims to present, in a unified manner, results which are valid on both the algebras of quaternions and coquaternions and, simultaneously, call the attention to the main differences between these two algebras. The rings of one-sided polynomials over each of these algebras are studied and some important differences in what concerns the structure of the set of their zeros are remarked. Examples illustrating this different behavior of the zero-sets of quaternionic and coquaternionic polynomials are also presented.
\end{abstract}

\section{Introduction}

Quaternions, introduced in 1843 by the Irish mathematician William Rowan Hamilton (1805-1865) as a generalization of complex numbers[11], have become a powerful tool for modeling and solving problems in classical fields of mathematics, engineering and physics[19]. One of the most widespread application of quaternions is in computer animation, where they are used to represent transformations of orientations of graphical objects [29]. The geometric and physical applications of quaternions require solving quaternion polynomial equations; see, e.g.,[1]. The literature on quaternion polynomial root-finding reveals a recent growing interest on this subject; see e.g. [7, 8, 12, 13, 16, 20, 25, 28, 27].

At about the same time Hamilton discovered the non-commutative algebra of quaternions, James Cockle (1819-1895) introduced another four-dimensional hypercomplex real algebra, the algebra of real coquaternions [6]. Probably due to the fact that this is a non-division algebra, coquaternions have received much less attention than their "cousins" quaternions. There are, however, some studies related to geometric applications of coquaternions ${ }^{1}$ [17],[23] and [24]. In addition, the relation between coquaternions and complexified mechanics is discussed in [5]. In what concerns coquaternionic polynomials, the bibliography is very scarce. The most relevant references on this subject are $[14,15,22]$ and [26].

The main purpose of this paper is to present, in a unified manner, results which are valid in both the algebras of quaternions and coquaternions and, at the same time, point out some important differences between these algebras. We also study one-sided polynomials defined over these two algebras, emphasizing the differences that may occur in the structure of their zero-sets.

\footnotetext{
${ }^{1}$ Also known, in the literature, as split-quaternions, para-quaternions, anti-quaternions or hyperbolic quaternions.
} 
The rest of the paper is organized as follows. Section 2 contains a revision of the main definitions and results on the algebras of quaternions and coquaternions. Section 3 is dedicated to the rings of (one-sided) polynomials over these two algebras and discusses, in particular, the main differences of the structure of the sets of zeros of these polynomial rings. Finally, Sect. 4 contains carefully chosen examples illustrating some of the conclusions contained in Sect. 3.

\section{The Algebras of Quaternions and Coquaternions}

Let $\{1, \mathbf{i}, \mathbf{j}, \mathbf{k}\}$ be an orthonormal basis of the Euclidean vector space $\mathbb{R}^{4}$ with a product given according to the multiplication rules

$$
\mathbf{i}^{2}=\mathbf{j}^{2}=\mathbf{k}^{2}=-1, \quad \mathbf{i j}=-\mathbf{j i}=\mathbf{k} .
$$

This non-commutative product generates the well known algebra of real quaternions, which we will denote by $\mathbb{H}$.

The algebra of real coquaternions, which we will denote by $\mathbb{H}_{\text {coq }}$, is generated by the product given according to the following rules

$$
\mathbf{i}^{2}=-1, \mathbf{j}^{2}=\mathbf{k}^{2}=1, \quad \mathbf{i j}=-\mathbf{j i}=\mathbf{k} .
$$

In what follows, since some of the results are valid in both algebras, we will use $\mathscr{H}$ to refer to one of the algebras $\mathbb{H}$ or $\mathbb{H}_{\text {coq. }}$.

We will embed the space $\mathbb{R}^{4}$ in $\mathscr{H}$ by identifying the element $q=\left(q_{0}, q_{1}, q_{2}, q_{3}\right)$ in $\mathbb{R}^{4}$ with the element $q=q_{0}+q_{1} \mathbf{i}+q_{2} \mathbf{j}+q_{3} \mathbf{k}$ in $\mathscr{H}$. Thus, throughout the paper, we will not distinguish an element in $\mathbb{R}^{4}$ and the corresponding quaternion/coquaternion, unless we need to stress the context.

If $p=p_{0}+p_{1} \mathbf{i}+p_{2} \mathbf{j}+p_{3} \mathbf{k}$ and $q=q_{0}+q_{1} \mathbf{i}+q_{2} \mathbf{j}+q_{3} \mathbf{k}$ are two given quaternions, then

$$
\begin{aligned}
p q=p_{0} q_{0}- & p_{1} q_{1}-p_{2} q_{2}-p_{3} q_{3}+\left(p_{0} q_{1}+p_{1} q_{0}+p_{2} q_{3}-p_{3} q_{2}\right) \mathbf{i} \\
& +\left(p_{0} q_{2}-p_{1} q_{3}+p_{2} q_{0}+p_{3} q_{1}\right) \mathbf{j}+\left(p_{0} q_{3}+p_{1} q_{2}-p_{2} q_{1}+p_{3} q_{0}\right) \mathbf{k}
\end{aligned}
$$

whilst, if $p$ and $q$ are coquaternions, we have

$$
\begin{aligned}
p q=p_{0} q_{0}- & p_{1} q_{1}+p_{2} q_{2}+p_{3} q_{3}+\left(p_{0} q_{1}+p_{1} q_{0}-p_{2} q_{3}+p_{3} q_{2}\right) \mathbf{i} \\
& +\left(p_{0} q_{2}-p_{1} q_{3}+p_{2} q_{0}+p_{3} q_{1}\right) \mathbf{j}+\left(p_{0} q_{3}+p_{1} q_{2}-p_{2} q_{1}+p_{3} q_{0}\right) \mathbf{k} .
\end{aligned}
$$

Given $q=q_{0}+q_{1} \mathbf{i}+q_{2} \mathbf{j}+q_{3} \mathbf{k} \in \mathscr{H}$, its conjugate $\bar{q}$ is defined as $\bar{q}=q_{0}-q_{1} \mathbf{i}-q_{2} \mathbf{j}-q_{3} \mathbf{k}$; the number $q_{0}$ is called the real part of $q$ and denoted by $\operatorname{Re} q$ and the vector part of $q$, denoted by $\operatorname{Vec} q$, is given by $\operatorname{Vec} q=q_{1} \mathbf{i}+q_{2} \mathbf{j}+q_{3} \mathbf{k}$.

We will identify the set of elements in $\mathscr{H}$ whose vector part is zero with the set $\mathbb{R}$ of real numbers. We will also consider three particularly important subspaces of dimension two of $\mathscr{H}$, usually called the canonical planes or cycle planes. The first is $\{q \in \mathscr{H}: q=a+b \mathbf{i}, a, b \in \mathbb{R}\}$ which, naturally, we identify with the complex plane $\mathbb{C}$; the second, which we denote by $\mathbb{P}$ and whose elements are usually called perplex numbers is given by $\mathbb{P}=\{q \in \mathscr{H}: q=a+b \mathbf{j}, a, b \in \mathbb{R}\}$ and the third, denoted by $\mathbb{D}$, is the subspace of the so-called dual numbers, $\mathbb{D}=\{q \in \mathscr{H}: q=a+b(\mathbf{i}+\mathbf{j}), a, b \in \mathbb{R}\}$; see e.g.[3].

We will denote by $\mathrm{t}(q)$ and call trace of $q$ the quantity given by

$$
\mathrm{t}(q)=q+\bar{q}=2 \operatorname{Re} q
$$

and will call determinant of $q$ and denote by $\mathrm{d}(q)$ the quantity given by $\mathrm{d}(q)=q \bar{q}$. We have

$$
\mathrm{d}(q)= \begin{cases}q_{0}^{2}+q_{1}^{2}+q_{2}^{2}+q_{3}^{2}, & \text { if } q \in \mathbb{H}, \\ q_{0}^{2}+q_{1}^{2}-q_{2}^{2}-q_{3}^{2}, & \text { if } q \in \mathbb{H}_{\mathrm{coq}} .\end{cases}
$$

Remark 1. Observe that, for a quaternion $q, \mathrm{~d}(q)$ is always a non-negative quantity and moreover $\mathrm{d}(q)=0$ iff $q=0$. In this case, the square root of $\mathrm{d}(q)$ is called the norm of $q$ and denoted by $|q|^{2}$

\footnotetext{
${ }^{2}$ This coincides with the Euclidean norm of the 4 -vector $\left(q_{0}, q_{1}, q_{2}, q_{3}\right)$.
} 
The following proposition lists some properties of quaternions/coquaternions which are easily verified; see e.g. [2] and [10].

Proposition 1. Given $p, q \in \mathscr{H}$ and $\alpha \in \mathbb{R}$, we have:

1. $q \in \mathbb{R} \Longleftrightarrow \bar{q}=q$

2. $\overline{\bar{q}}=q$

3. $\overline{p+q}=\bar{p}+\bar{q}$

4. $\overline{p q}=\bar{q} \bar{p}$

5. $\operatorname{Re} q=\operatorname{Re} \bar{q}$

6. $\operatorname{Re}(p q)=\operatorname{Re}(q p)$

7. $\mathrm{d}(q)=(\operatorname{Re} q)^{2}+\mathrm{d}(\operatorname{Vec} q)$

8. $\mathrm{d}(q)=\mathrm{d}(\bar{q})$

9. $\mathrm{d}(\alpha \mathrm{q})=\alpha^{2} \mathrm{~d}(q)$

10. $\mathrm{d}(p q)=\mathrm{d}(q p)=\mathrm{d}(p) \mathrm{d}(q)$

11. $q$ commutes with any other element in $\mathscr{H}$ if and only if $q \in \mathbb{R}$ (i.e. the center of $\mathscr{H}$ is $\mathbb{R}$ ).

We also have the following result, very simple to prove.

Proposition 2. An element $q \in \mathcal{H}$ is invertible if and only if $\mathrm{d}(q) \neq 0$. In that case, we have $q^{-1}=\frac{\bar{q}}{\mathrm{~d}(q)}$.

A non-invertible element $q \in \mathscr{H}$ is also called a singular element. Since, as observed in Remark 1 , the only quaternion with a zero determinant is $q=0$, we immediately conclude that all non-zero quaternions are invertible, i.e. $\mathbb{H}$ is a division algebra. In the case of coquaternions, there are, however, non-zero singular elements, such as, for example, the coquaternion $q=1+\mathbf{i}+\mathbf{j}+\mathbf{k}$.

It can be shown that $q \neq 0$ is singular if and only if $q$ is a zero-divisor, i.e. there exist $p, r \in \mathbb{H}_{\text {coq }}$ such that $q p=r q=0$.

Definition 1. We say that an element $q \in \mathscr{H}$ is similar to another element $p \in \mathscr{H}$, and write $q \sim p$, if there exists an invertible $h \in \mathscr{H}$ such that $q=h^{-1} p h$.

This is an equivalence relation in $\mathscr{H}$ (hence, we can simply say that two elements $p, q \in \mathscr{H}$ are similar), partitioning $\mathscr{H}$ in the so-called similarity classes. We denote by $[q]$ the similarity class containing a given element $q \in \mathscr{H}$. The next proposition shows that the equivalence classes of real elements reduce to a single element.

Proposition 3. Let $q \in \mathscr{H}$. Then

$$
[q]=\{q\} \Longleftrightarrow q \in \mathbb{R} .
$$

Proof. If $q \in \mathbb{R}$ and $p \sim q$, then $p=h^{-1} q h=q h^{-1} h=q$. On the other hand, if $[q]=\{q\}$, we have that $h^{-1} q h=q$ or, equivalently, $q h=h q$, for all invertible $h$. This means, in particular, that $q$ must commute with $\mathbf{i}, \mathbf{j}$ and $\mathbf{k}$, which can only be true if $q \in \mathbb{R}$.

In what follows, given an element $q=q_{0}+q_{1} \mathbf{i}+q_{2} \mathbf{j}+q_{3} \mathbf{k} \in \mathscr{H}$, we will use $\operatorname{dv}(q)$ to denote the determinant of the vector part of $q$, i.e. $\operatorname{dv}(q)=\mathrm{d}(\operatorname{Vec} q)$. Note that:

- for $q \in \mathbb{H}, \operatorname{dv}(q)=q_{1}^{2}+q_{2}^{2}+q_{3}^{2}$; hence $\operatorname{dv}(q)$ is always a non-negative quantity and $\operatorname{dv}(q)=0$ if and only if $q \in \mathbb{R}$;

- for $q \in \mathbb{H}_{\mathrm{coq}}, \operatorname{dv}(q)=q_{1}^{2}-q_{2}^{2}-q_{3}^{2}$; hence $\operatorname{dv}(q)$ can be negative, null or positive; moreover, $\operatorname{dv}(q)=0$ does not imply $q \in \mathbb{R}$. 
Definition 2. We say that two elements $p, q \in \mathscr{H}$ are $q u a s i$-similar, and write $p \approx q$, if and only if they satisfy the following conditions

$$
\operatorname{Re} p=\operatorname{Re} q \text { and } \operatorname{dv}(p)=\operatorname{dv}(q) \text {. }
$$

This is an equivalence relation in $\mathscr{H}$, partitioning $\mathscr{H}$ in the so-called quasi-similarity classes. We denote by $\llbracket q \rrbracket$ the quasi-similarity class containing a given element $q \in \mathscr{H}$.

Remark 2. The above definition was introduced, for the case of coquaternions, by Janovská and Opfer[15], but a different notation was used.

Note that, since $\mathrm{d}(q)=(\operatorname{Re} q)^{2}+\mathrm{dv}(q)$, the conditions (4) defining quasi-similarity are equivalent to the conditions

$$
\operatorname{Re} p=\operatorname{Re} q \quad \text { and } \quad \mathrm{d}(p)=\mathrm{d}(q) \text {. }
$$

We will now show that the concepts of quasi-similarity and similarity coincide for any two quaternions and also for any two non-real coquaternions. Only for the case where one of the coquaternions is real and the other is non-real, the concepts of quasi-similarity and similarity do not coincide.

Theorem 1. [4, Lemma 3] Let $q=q_{0}+q_{1} \mathbf{i}+q_{2} \mathbf{j}+q_{3} \mathbf{k} \in \mathbb{H}$. Then, $q$ is similar to the complex number $q_{0}+\sqrt{\operatorname{dv}(q)} \mathbf{i}$, i.e. $[q]=\left[q_{0}+\sqrt{\operatorname{dv}(q)} \mathbf{i}\right]$.

Corollary 1. Two quaternions $p, q \in \mathbb{H}$ are similar if and only if they are quasi-similar.

Proof. If $p$ and $q$ are similar, then it is an immediate consequence of 6 . and 10 . in Proposition 1 that they satisfy (5). The fact that two elements $p, q \in \mathbb{H}$ satisfying (4) are similar is an immediate consequence of the previous theorem, having in mind that similarity is an equivalence relation.

From the previous corollary, we have, for any $q=q_{0}+\mathrm{q}_{1} \mathbf{i}+\mathrm{q}_{2} \mathbf{j}+\mathrm{q}_{3} \mathbf{k} \in \mathbb{H}$

$$
\begin{aligned}
{[q]=\llbracket q \rrbracket } & =\{p \in \mathbb{H}: \operatorname{Re} p=\operatorname{Re} q \text { and } \operatorname{dv}(p)=\operatorname{dv}(q)\} \\
& =\left\{p_{0}+p_{1} \mathbf{i}+p_{2} \mathbf{j}+p_{3} \mathbf{k}: p_{0}=q_{0} \text { and } p_{1}^{2}+p_{2}^{2}+p_{3}^{2}=\operatorname{dv}(q)\right\}
\end{aligned}
$$

This shows that the similarity class of a quaternion $q=q_{0}+q_{1} \mathbf{i}+q_{2} \mathbf{j}+q_{3} \mathbf{k}$ can be identified with a sphere in the hyperplane $\left\{\left(x_{0}, x_{1}, x_{2}, x_{3}\right) \in \mathbb{R}^{4}: x_{0}=q_{0}\right\}$. This sphere is centered at the point $\left(q_{0}, 0,0,0\right)$ and has radius $\sqrt{\operatorname{dv}(q)}$, reducing to a single point when $\operatorname{dv}(q)=0$ i.e., when $q \in \mathbb{R}$.

Theorem 2. Let $q=q_{0}+q_{1} \mathbf{i}+q_{2} \mathbf{j}+q_{3} \mathbf{k} \in \mathbb{H}_{\text {coq }}$ be a non-real coquaternion. Then:

(i) if $\operatorname{dv}(q)>0, q$ is similar to the complex number $q_{0}+\sqrt{\mathrm{dv}(q)} \mathbf{i}$, i.e. $[q]=\left[q_{0}+\sqrt{\operatorname{dv}(q)} \mathbf{i}\right]$

(ii) if $\operatorname{dv}(q)<0, \quad q$ is similar to the perplex number $q_{0}+\sqrt{-\mathrm{dv}(q)} \mathbf{j}$, i.e. $[q]=\left[q_{0}+\sqrt{-\operatorname{dv}(q)} \mathbf{j}\right]$;

(iii) if $\operatorname{dv}(q)=0, q$ is similar to the dual number $q_{0}+\mathbf{i}+\mathbf{j}$, i.e. $[q]=\left[q_{0}+\mathbf{i}+\mathbf{j}\right]$.

Proof. In each case, we will indicate how to choose an invertible $h \in \mathbb{H}_{\text {coq }}$ such that $h^{-1} q h$ has the specified form.

(i) Naturally, if $q=q_{0}+q_{1}$ i with $q_{1}>0$, then $q_{1}=\sqrt{\operatorname{dv}(q)}$ and there is nothing to prove. Otherwise, we can take

$$
h= \begin{cases}\mathbf{j}, & \text { if } q_{2}^{2}+q_{3}^{2}=0, \\ \left(q_{1}+\sqrt{\mathrm{dv}(q)}\right)-q_{3} \mathbf{j}+q_{2} \mathbf{k}, & \text { if } \quad q_{2}^{2}+q_{3}^{2} \neq 0 .\end{cases}
$$

Note that, for $h=\left(q_{1}+\sqrt{\operatorname{dv}(q)}\right)-q_{3} \mathbf{j}+q_{2} \mathbf{k}$, we have

$$
\mathrm{d}(h)=\left(q_{1}+\sqrt{\mathrm{dv}(q)}\right)^{2}-q_{3}^{2}-q_{2}^{2}=2 \sqrt{\operatorname{dv}(q)}\left(\sqrt{\operatorname{dv}(q)}+q_{1}\right) .
$$


Since

$$
q_{2}^{2}+q_{3}^{2} \neq 0 \Rightarrow \operatorname{dv}(q) \neq q_{1}^{2} \Rightarrow \sqrt{\operatorname{dv}(q)}+q_{1} \neq 0,
$$

the condition $q_{2}^{2}+q_{3}^{2} \neq 0$ guarantees that $\mathrm{d}(h) \neq 0$, i.e. that $h$ is an invertible element. Moreover, it is easy to verify that $q h=h\left(q_{0}+\sqrt{\operatorname{dv}(q)} \mathbf{i}\right)$.

(ii) Similarly to the previous case, if $q=q_{0}+q_{2} \mathbf{j}$ with $q_{2}>0$, then $q_{2}=\sqrt{-\operatorname{dv}(q)}$, so there is nothing to prove. Otherwise, we can consider

$$
h= \begin{cases}\mathbf{i}, & \text { if } q_{1}^{2}+q_{3}^{2}=0, \\ q_{1}-q_{3} \mathbf{j}+\left(q_{2}-\sqrt{-\operatorname{dv}(q)}\right) \mathbf{k}, & \text { if } \quad q_{1}^{2}+q_{3}^{2} \neq 0 \text { and } q_{2} \leq 0, \\ \left(q_{2}+\sqrt{-\operatorname{dv}(q)}\right)+q_{3} \mathbf{i}+q_{1} \mathbf{k}, & \text { if } \quad q_{1}^{2}+q_{3}^{2} \neq 0 \text { and } q_{2}>0 .\end{cases}
$$

In a manner similar to the previous case, one can verify that the coquaternions $h$ given by the above formulas are invertible and are such that $q h=h\left(q_{0}+\sqrt{-\operatorname{dv}(q)} \mathbf{j}\right)$.

(iii) We can take

$$
h= \begin{cases}\left(1+q_{1}\right)-q_{3} \mathbf{j}-\left(1-q_{2}\right) \mathbf{k}, & \text { if } q_{1}+q_{2} \neq 0, \\ \left(1+q_{1}\right) \mathbf{i}+\left(1-q_{1}\right) \mathbf{j}, & \text { if } q_{1}+q_{2}=0 .\end{cases}
$$

Once more, it is easy to verify that the coquaternions $h$ satisfy $q h=h(1+\mathbf{i}+\mathbf{j})$ and, under the conditions stated, are invertible.

Remark 3. The explicit expressions for $h$ in the cases (i) and (ii), here included for completeness, can be seen in [17].

Corollary 2. Two non-real coquaternions $p, q \in \mathbb{H}_{\mathrm{coq}}$ are similar if and only if they are quasi-similar.

Proof. The proof is totally analogous to the proof of Corollary 1, now making use of the previous theorem.

For a given element $q=q_{0}+q_{1} \mathbf{i}+q_{2} \mathbf{j}+q_{3} \mathbf{k} \in \mathbb{H}_{\text {coq }}$, we have

$$
\begin{aligned}
\llbracket q \rrbracket & =\left\{p \in \mathbb{H}_{\text {coq }}: \operatorname{Re}(p)=\operatorname{Re}(q) \text { and } \operatorname{dv}(p)=\operatorname{dv}(q)\right\} \\
& =\left\{p_{0}+p_{1} \mathbf{i}+p_{2} \mathbf{j}+p_{3} \mathbf{k}: p_{0}=q_{0} \text { and } p_{1}^{2}-p_{2}^{2}-p_{3}^{2}=\operatorname{dv}(q)\right\}
\end{aligned}
$$

Hence, the quasi-similarity class of $q$ can be identified with an hyperboloid in the hyperplane $\left\{\left(x_{0}, x_{1}, x_{2}, x_{3}\right) \in\right.$ $\left.\mathbb{R}^{4}: x_{0}=q_{0}\right\}$. This will be:

- an hyperboloid of two sheets, if $\operatorname{dv}(q)>0$; in this case

$$
\llbracket q \rrbracket=[q]=\left[q_{0}+\sqrt{\operatorname{dv}(q)} \mathbf{i}\right] ;
$$

- an hyperboloid of one sheet, if $\operatorname{dv}(q)<0$; in this case

$$
\llbracket q \rrbracket=[q]=\left[q_{0}+\sqrt{-\operatorname{dv}(q)} \mathbf{j}\right] ;
$$

- a degenerate hyperboloid (i.e. a cone), if $\operatorname{dv}(q)=0$; in this case,

$$
\llbracket q \rrbracket=\llbracket q_{0} \rrbracket \quad \text { and } \quad[q]=\left\{\begin{array}{ll}
\left\{q_{0}\right\}, & \text { if } q \in \mathbb{R} \\
{\left[q_{0}+\mathbf{i}+\mathbf{j}\right]=\llbracket q_{0} \rrbracket \backslash\left\{q_{0}\right\},} & \text { if } q \notin \mathbb{R}
\end{array} ;\right.
$$

see Fig. 1. 


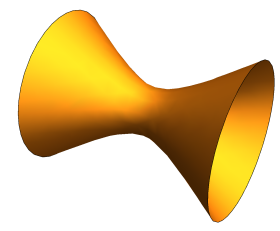

(a)

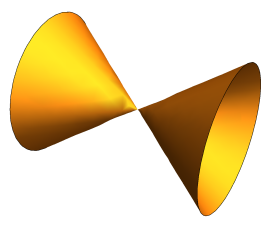

(b)

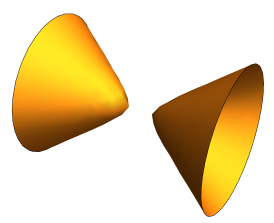

(c)

Figure 1: Plots, in the hyperplane $\left\{\left(x_{0}, x_{1}, x_{2}, x_{3}\right) \in \mathbb{R}^{4}: x_{0}=q_{0}\right\}$, of the quasi-similarity class of a coquaternion $q$. (a) $\operatorname{dv}(q)<0 ;(b) \operatorname{dv}(q)=0 ;(c) \operatorname{dv}(q)>0$.

\section{One-Sided Polynomials}

We denote by $\mathscr{H}[x]$ the set of polynomials of the form

$$
P(x)=c_{n} x^{n}+c_{n-1} x^{n-1}+\cdots+c_{1} x+c_{0}, c_{i} \in \mathscr{H},
$$

i.e., the set of polynomials whose coefficients are only located on the left of the variable, with the addition and multiplication of such polynomials defined as in the commutative case, where the variable is assumed to commute with the coefficients. This is a ring, referred to as the ring of (left) one-sided, unilateral or simple polynomials in $\mathscr{H} .^{3}$

As usual, if $c_{n} \neq 0$, we will say that the degree of the polynomial $P(x)$ is $n$ and refer to $c_{n}$ as the leading coefficient of the polynomial. When $c_{n}=1$, we say that $P(x)$ is monic. If the coefficients $c_{i}$ in $(6)$ are real, then we say that $P(x)$ is a real polynomial.

The evaluation map at a given element $q \in \mathscr{H}$, defined, for the polynomial $P(x)$ given by (6), by $P(q)=c_{n} q^{n}+c_{n-1} q^{n-1}+\cdots+c_{1} q+c_{0}$, is not a homomorphism from the ring $\mathscr{H}[x]$ into $\mathscr{H}$. In fact, given two polynomials $L(x), R(x) \in \mathbb{H}[x]$, in general we do not have $(L R)(q)=L(q) R(q)$.

Remark 4. Since all the polynomials considered will be in the indeterminate $x$, we will usually omit the reference to this variable and write simply $P$ when referring to an element $P(x) \in \mathscr{H}[x]$, the expression $P(q)$ being preferably reserved for the evaluation of $P$ at a specific value $q \in \mathscr{H}$.

We say that an element $q \in \mathscr{H}$ is a zero (or a root) of a polynomial $P$, if $P(q)=0$, and we use the notation $Z(P)$ to denote the zero-set of $P$, i.e. the set of all the zeros of $P$.

The next theorem has some results concerning the evaluation, at a given element $q \in \mathscr{H}$, of the product of two polynomials.

Theorem 3. Let $L(x)=\sum_{i=0}^{n} a_{i} x^{i}$ and $R(x)=\sum_{j=0}^{m} b_{j} x^{j}$ be two given polynomials in $\mathscr{H}[x], P(x)=$ $L(x) R(x)$ and $q \in \mathscr{H}$. Then, we have:

(i) $P(q)=\sum_{i=0}^{n} a_{i} R(q) q^{i}$.

(ii) If $R(q)=0$, then $P(q)=0$.

(iii) If $R(q)$ is non-singular, then $P(q)=L(\tilde{q}) R(q)$, where $\tilde{q}=R(q) q(R(q))^{-1}$.

(iv) If $L(x)$ is a real polynomial, then $(L R)(q)=(R L)(q)=R(q) L(q)$.

(v) If $q \in \mathbb{R}$, then $(L R)(q)=L(q) R(q)$.

\footnotetext{
${ }^{3}$ Right one-sided polynomials are defined in an analogous manner, by considering the coefficients on the right of the variable; all the results for left one-sided polynomials have corresponding results for right one-sided polynomials and hence we restrict our study to polynomials of the first type.
} 
Proof. The proofs of (i)-(iii) are very simple adaptations of the corresponding proofs for similar results for polynomials over a division ring given in [18, Proposition (16.2)], noting that the condition " $R(q)$ is nonsingular" assumed on (iii) plays the same role of the condition $R(q) \neq 0$ in the referred proposition in [18].

The results (iv) $-(\mathrm{v})$ follow easily from the definition of the product of polynomials and the fact that real numbers commute with any element in $\mathscr{H}$.

As an immediate consequence of this theorem, we have the following result.

Corollary 3. Let $L(x)$ and $R(x)$ be two given polynomials in $\mathscr{H}[x], P(x)=(L R)(x)$ and $q \in \mathscr{H}$. If $R(q)$ is non-singular, then $q$ is a zero of $P$ if and only if $R(q) q(R(q))^{-1}$ is a zero of $L$.

Remark 5. Since, in the case of quaternions, all non-zero elements are non-singular, the condition " $R(q)$ is non-singular" in Theorem 3-(iii) and in Corollary 3 can, in this case, be replaced by $R(q) \neq 0$.

Definition 3. Given an element $q \in \mathscr{H}$, the characteristic polynomial of $q$, denoted $\Psi_{q}(x)$, is the polynomial defined by

$$
\Psi_{q}(x)=(x-q)(x-\bar{q})=(x-\bar{q})(x-q)=x^{2}-\mathrm{t}(q) x+\mathrm{d}(q) .
$$

Note that $\Psi_{q}$ is a monic quadratic real polynomial and also that we have

$$
\Psi_{q}=\Psi_{q^{\prime}} \Longleftrightarrow \llbracket q \rrbracket=\llbracket q^{\prime} \rrbracket \Longleftrightarrow q^{\prime} \in \llbracket q \rrbracket .
$$

Equation (8) shows that $\Psi_{q}$ is an invariant of the quasi-similarity class of $q .{ }^{4}$

Remark 6. Having in mind the previous observation, it would probably be more appropriate to say that the polynomial given by (7) is the characteristic polynomial of the quasi-similarity class $\llbracket q \rrbracket$ and to denote it by $\Psi_{\llbracket q \rrbracket}$. However, for a question of simplicity, we will stick to the denomination and notation introduced in Definition 3.

Let $\Psi_{q}$ be the characteristic polynomial of a given element $q \in \mathscr{H}$. The discriminant of $\Psi_{q}$ is

$$
\Delta=(\mathrm{t}(q))^{2}-4 \mathrm{~d}(q)=4(\operatorname{Re} q)^{2}-4\left((\operatorname{Re} q)^{2}+\operatorname{dv}(q)\right)=-4 \operatorname{dv}(q) .
$$

Thus:

1. In the quaternionic case, $\Delta$ will never be positive, being zero if and only if $q \in \mathbb{R}$. Hence, the characteristic polynomial of any non-real quaternion is an irreducible (over the reals) monic quadratic polynomial. The characteristic polynomial of $q \in \mathbb{R}$ is, naturally, the polynomial $(x-q)^{2}$.

2. In the coquaternionic case, $\Delta$ will be negative, null or positive, depending on whether $\operatorname{dv}(q)>0, \operatorname{dv}(q)=$ 0 or $\operatorname{dv}(q)<0$, respectively. This means that $\Psi_{q}$ will be:

- an irreducible polynomial, if $\operatorname{dv}(q)>0$;

- a polynomial of the form $(x-r)^{2}, r \in \mathbb{R}$, if $\operatorname{dv}(q)=0$;

- a polynomial of the form $\left(x-r_{1}\right)\left(x-r_{2}\right)$ with $r_{1}, r_{2} \in \mathbb{R}, r_{1} \neq r_{2}$, if $\operatorname{dv}(q)<0$.

The next theorem lists some properties involving the zero-set of the characteristic polynomial of an element $q \in \mathscr{H}$.

Theorem 4. Let $\Psi_{q}$ be the characteristic polynomial of a given element $q \in \mathscr{H}$. Then:

(i) $\llbracket q \rrbracket \subseteq Z\left(\Psi_{q}\right)$

(ii) If $\mathrm{dv}_{q} \geq 0, Z\left(\Psi_{q}\right)=\llbracket q \rrbracket$.

(iii) If $\mathrm{dv}_{q}<0$, then $\Psi_{q}(x)=\left(x-r_{1}\right)\left(x-r_{2}\right)$ for $r_{1}, r_{2} \in \mathbb{R}, r_{1} \neq r_{2}$ and $Z\left(\Psi_{q}\right)=\llbracket q \rrbracket \cup\left\{r_{1}, r_{2}\right\}$.

\footnotetext{
${ }^{4}$ Recall that, in the case of quaternions, quasi-similarity and similarity classes coincide, so, in that case, we can also say that $\Psi_{q}$ is an invariant of $[q]$
} 
Proof. Let $u$ be an arbitrary element in $\llbracket q \rrbracket$. From the relation $u=\mathrm{t}(u)-\bar{u}$ (see (1)), we obtain

$$
u^{2}=(\mathrm{t}(u)-\bar{u}) u=\mathrm{t}(u) u-\mathrm{d}(u),
$$

or

$$
u^{2}-\mathrm{t}(u) u+\mathrm{d}(u)=0 .
$$

This shows that $\Psi_{q}(u)=0$, hence establishing (i).

We now prove (ii)-(iii). Let then $z$ be a zero of $\Psi_{q}$, i.e., let $z$ satisfy $z^{2}-\mathrm{t}(q) z+\mathrm{d}(q)=0$; since $z$ is also a zero of its characteristic polynomial (by (i)), we also have $z^{2}-\mathrm{t}(z) z+\mathrm{d}(z)=0$ and so we obtain $(\mathrm{t}(z)-\mathrm{t}(q)) z+\mathrm{d}(q)-\mathrm{d}(z)=0$, or $(\mathrm{t}(z)-\mathrm{t}(q))(\operatorname{Re} z+\operatorname{Vec} z)+\mathrm{d}(q)-\mathrm{d}(z)=0$, which means that we must have

$$
\left\{\begin{array}{l}
(\mathrm{t}(z)-\mathrm{t}(q)) \operatorname{Vec} z=0 \\
(\mathrm{t}(z)-\mathrm{t}(q)) \operatorname{Re} z+\mathrm{d}(q)-\mathrm{d} z=0
\end{array} .\right.
$$

If $z$ is non-real, i.e. if $\operatorname{Vec} z \neq 0$, we immediately obtain $\mathrm{t}(z)=\mathrm{t}(q)$ and $\mathrm{d}(z)=\mathrm{d}(q)$, showing that $z \in \llbracket q \rrbracket$. Hence, we conclude that all non-real roots of $\Psi_{q}$ belong to $\llbracket q \rrbracket$.

Let us now see what happens with the (possible) real zeros of $\Psi_{q}$. Recalling the description on the behavior of the characteristic polynomial $\Psi_{q}$ done previously, we see that this type of roots only occur if $\operatorname{dv}(q)=0$ or $\operatorname{dv}(q)<0$. In the first case, we have that $\Psi_{q}$ has a double real root $r$, but $\llbracket q \rrbracket=\llbracket r \rrbracket$, i.e., $r \in \llbracket q \rrbracket$ and so the result (ii) follows. When $\operatorname{dv}(q)<0^{5}$ we have that $\Psi_{q}(x)=\left(x-r_{1}\right)\left(x-r_{2}\right)$ has two real zeros $r_{1}$ and $r_{2}$ which do not belong to $\llbracket q \rrbracket$ (note that, in that case, $\operatorname{Re} q=\frac{r_{1}+r_{2}}{2} \neq r_{1}, r_{2}$ ), which establishes (iii).

The previous theorem shows us that, in what concerns the number of zeros, quaternionic and coquaternionic polynomials may behave very differently from polynomials in $\mathbb{C}[x]$, where, as is well known, a polynomial of degree $n$ has, at most, $n$ distinct zeros. In fact, the characteristic polynomial of an element $q \in \mathscr{H}$ (with $q$ nonreal, in the quaternionic case) gives us an example of a polynomial with an infinite number of zeros: a sphere, in the quaternionic case, and an hyperboloid or an hyperboloid and two extra zeros, in the coquaternonic case.

Although quaternionic and coquaternonic polynomials have this common feature - they both may have an infinite number of zeros - there is a very important difference between these two types of polynomials. In the case of quaternion polynomials, as proved in the pioneering paper by I. Niven[21], the Fundamental Theorem of Algebra is valid, i.e., every non-constant polynomial in $\mathbb{H}[x]$ has, at least, a zero in $\mathbb{H}$. However, this theorem is not valid in $\mathbb{H}_{\text {coq }}[x]$. In fact, as observed by Özdemir[22, Theorem 9-i.], since for a coquaternion $u$, we have $\mathrm{d}\left(u^{n}\right)=(\mathrm{d}(u))^{n}$ (see Proposition 1-10.)), any equation of the form $x^{n}-q=0$, with $n$ even and $q$ a coquaternion with negative determinant, does not have a solution. Other examples of coquaternion polynomials with no roots can be found in [15].

We now introduce the following definition for zeros of a polynomial in $\mathscr{H}[x]$.

Definition 4. Let $P \in \mathscr{H}[x]$ and let $z \in \mathscr{H}$ be a zero of $P$.

(i) If $\llbracket z \rrbracket$ contains no other zeros of $P$, then $z$ is said to be an isolated zero of $P$.

(ii) If $\llbracket z \rrbracket \subseteq Z(P)$, then $z$ is said to be:

- a spherical zero of $P$, if $\mathscr{H}=\mathbb{H}$;

- an hyperboloidal zero of $P$, if $\mathscr{H}=\mathbb{H}_{\text {coq }}$.

Remark 7. 1. The choice of the denominations, spherical, in the case of quaternionic polynomials and hyperboloidal, in the case of coquaternionic polynomials, is a natural one, having in mind the type of sets which are the quasi-similarity classes, in each case. The term hyperboloidal, which we adopt here, was the choice made by Pogoruy and Ramírez-Dagnino in [26]; in [15], the authors use the term hyperbolic instead of hyperboloidal, which, from our point of view, does not seem so appropriate.

2. In the quaternionic case, a real zero $z$, is by definition, an isolated zero, since, in this case $\llbracket z \rrbracket=[z]=\{z\}$. This is not necessarily true in the coquaternionic case. Example 2, given later, will clarify this assertion.

3. When a zero $z$ is spherical/hyperboloidal, sometimes we treat the whole class as a single zero and talk about the spherical/hyperboloidal zero $\llbracket z$.

\footnotetext{
${ }^{5}$ Recall that this can only happen in the case of coquaternions.
} 
Theorem 5. Let $P \in \mathscr{H}[x]$ and let $z \in \mathscr{H}$ be a zero of $P$. If $\llbracket z \rrbracket$ contains another zero $u$ of $P$ such that $z-u$ is nonsingular, then $\llbracket z \rrbracket \subseteq Z(P)$.

Proof. We first observe that, since the product of two polynomials in $\mathscr{H}[x]$ is defined in the usual manner, the Euclidean Division Algorithm to perform the division of two polynomials can be applied, provided that the leading coefficient of the divisor polynomial is a non-singular element in $\mathscr{H}$. We can thus divide $P(x)$ by the characteristic polynomial of $z$, i.e. by the quadratic monic polynomial $\Psi_{z}(x)=x^{2}-\mathrm{t}(z) x+\mathrm{d}(z)$, obtaining

$$
P(x)=Q(x) \Psi_{z}(x)+A+B x
$$

for some polynomial $Q(x)$ and values $A$ and $B$ which depend only on the coefficients $c_{i}$ of the polynomial $P$ and on the values $\mathrm{t}(z)$ and $\mathrm{d}(z)$. Hence, for all $w \in \llbracket z \rrbracket$, we have, $P(w)=A+B w$, since $\Psi_{z}(w)=0($ see Theorem 4-(i)). Because, by hypothesis, $z$ and $u$ are both zeros of $P$ in $\llbracket z \rrbracket$, it follows that we must have

$$
A+B z=0 \text { and } A+B u=0
$$

from where we obtain $B(u-z)=0$. The fact that $u-z$ is non-singular implies that $B=0$ which, from (10), leads to $A=0$ also. From (9), we then obtain $P(x)=Q(x) \Psi_{z}(x)$, allowing us to conclude that all the zeros of $\Psi_{z}$ are zeros of $P$; see Theorem 3-(ii). Since $\llbracket z \rrbracket \subseteq Z\left(\Psi_{z}\right)$ (Theorem 4-(i)), the result follows.

Remark 8. The result of Theorem 5 for the case of quaternionic polynomials was first established, by using totally different arguments, by Gordon and Motzkin [9]. For the coquaternionic case, a more similar proof to the one presented here was given in [15].

In the case of quaternionic polynomials, the condition $u-z$ non-singular can simply be replaced by $u-z \neq 0$ i.e., by $u \neq z$. This shows that, in the quaternionic case, a zero can only be of one of two types: isolated when its similarity class contains no other zeros - or spherical, if the class contains more than one zero. In particular, a (non-real) zero $z$ of $P$ is spherical if and only its conjugate $\bar{z}$ is also a zero of $P$. This is a very simple test one usually performs to distinguish an isolated zero from a spherical one.

In the case of coquaternionic polynomials, however, this kind of dichotomy does not hold anymore. In fact, it is possible to have a zero of a polynomial whose corresponding quasi-similarity class contains other zeros of the polynomial, but such that not all the elements in the class are zeros. Examples of such type of zeros will be given later.

Theorem 6. Let $P \in \mathscr{H}[x]$ be a real polynomial. If $z$ is a zero of $P$, then $[z] \subseteq Z(P)$.

Proof. See e.g. the proof of [30, Lemma 2.3.] for the quaternionic case or the analogous proof in [15, Theorem $1.2]$, where the result is stated for the coquaternionic case.

Corollary 4. Under the conditions of the previous theorem, if $z$ is a non-real zero of $P$, then the zero is spherical, in the quaternionic case, and hyperboloidal, in the coquaternionic case.

Proof. The conclusion, for the quaternionic case, is immediate, since $[z]=\llbracket z \rrbracket$. We now consider the coquaternionic case. Note that, in this case, the fact that $z$ is non-real does not guarantee that $\llbracket z \rrbracket=[z]$ (this does not hold when $\operatorname{dv}(z)=0)$.

Let $z=z_{0}+z_{1} \mathbf{i}+z_{2} \mathbf{j}+z_{3} \mathbf{k}$. Since $z$ is non-real, we have $z_{i} \neq 0$ for at least one $i \in\{1,2,3\}$. Assume, for example, that $z_{1} \neq 0$ (the proof is analogous in other cases) and consider the coquaternion $u=z_{0}-z_{1} \mathbf{i}+z_{2} \mathbf{j}+z_{3} \mathbf{k}$. Then, $u \in \llbracket z \rrbracket$ and both $u$ and $z$ are non-real; this means that $u \sim z$, i.e., $u \in[z]$ and so, from the previous theorem, $u$ is a zero of $P$; on the other hand, $\mathrm{d}(z-u)=\mathrm{d}\left(2 z_{1} \mathbf{i}\right)=4 z_{1}^{2} \neq 0$. Theorem 5 guarantees that the zero is hyperboloidal.

Remark 9. The conclusion of the previous corollary is stated in [15, Example 3.6] as being an immediate consequence of Theorem 6. However, as explained before, in the coquaternionic case, the fact that $\llbracket z \rrbracket \subseteq Z(P)$ is not obvious and needs to be proved. 


\section{Examples}

In this section, we present simple examples illustrating some of the results on the zeros of polynomials in $\mathbb{H}[x]$ and $\mathbb{H}_{\text {coq }}[x]$ previously discussed.

We first summarize the main differences which can exist between quaternionic and coquaternionic polynomials, in what concerns their zeros.

1. The Fundamental Theorem of Algebra is valid in $\mathbb{H}[x]$, i.e., every non-constant quaternionic polynomial has, at least, a zero in $\mathbb{H}$, but is no longer valid in $\mathbb{H}_{\text {coq }}[x]$.

2. A real zero of a quaternionic polynomial is always an isolated zero, but this is not necessarily the case for coquaternionic polynomials.

3. A zero $z$ of a quaternionic polynomial $P$ can be only of two types: isolated, if $z$ is the only zero of $P$ in $\llbracket z \rrbracket$, or spherical, if $\llbracket z \rrbracket$ contains more than one zero of $P$, in which case all the elements in the sphere $\llbracket z \rrbracket$ are zeros of $P$. In the coquaternionic case, zeros of other type may appear for a given polynomial $P$ : zeros $z$ such that $\llbracket z \rrbracket$ contains other zeros of $P$, but such that not all the class $\llbracket z \rrbracket$ is formed by zeros of $P$.

Example 1. As observed before, if $n$ is even and $q$ is a coquaternion with a negative determinant, then the polynomial $P(x)=x^{n}-q$ has no coquaternionic roots. Hence, for example, the polynomial $P_{1}(x)=x^{2}-\mathbf{j}$ in $\mathbb{H}_{\text {coq }}[x]$ has no zeros.

Note that if we view $P_{1}$ as a quaternionic polynomial, then $P_{1}$ has two isolated zeros: $x_{1}=\frac{\sqrt{2}}{2}+\frac{\sqrt{2}}{2} \mathbf{j}$ and $x_{2}=-\frac{\sqrt{2}}{2}-\frac{\sqrt{2}}{2} \mathbf{j}$.

Example 2. Consider the real polynomial

$$
P_{2}(x)=(x-1)(x-2)=x^{2}-3 x+2 .
$$

The only quaternionic zeros of this polynomial are the isolated zeros $x_{1}=1$ and $x_{2}=2$.

However, if we consider $P_{2}$ as a polynomial in $\mathbb{H}_{\text {coq }}[x]$, then, since $P_{2}(x)=\Psi_{q}(x)$ with $q$ the coquaternion $q=\frac{3}{2}+\frac{1}{2} \mathbf{j}$, Theorem 4 allows us to conclude that $P_{2}$ has, apart from the two isolated zeros $x_{1}=1$ and $x_{2}=2$, an hyperboloid of zeros: the quasi-similarity class $\llbracket \frac{3}{2}+\frac{1}{2} \mathbf{j} \rrbracket$.

Example 3. Let

$$
P_{3}(x)=(x-2)\left(x-\left(\frac{3}{2}+\frac{1}{2} \mathbf{j}\right)\right)=x^{2}-\left(\frac{7}{2}+\frac{1}{2} \mathbf{j}\right) x+3+\mathbf{j} .
$$

If we consider the polynomial $P_{3}$ as an element of $\mathbb{H}[x]$, then $P_{3}$ has only two isolated roots: $x_{1}=2$ and $x_{2}=\frac{3}{2}+\frac{1}{2} \mathbf{j}$.

Considering $P_{3}$ as a polynomial in $\mathbb{H}_{\text {coq }}[x]$, a simple verification shows that any coquaternion in the set

$$
\mathscr{Z}_{1}=\{2+\alpha \mathbf{i}-\alpha \mathbf{k}: \alpha \in \mathbb{R}\} \varsubsetneqq \llbracket 2 \rrbracket
$$

is a zero of $P_{3}$. However, not all the elements in the quasi-similarity class $\llbracket 2 \rrbracket$ are zeros of $P_{3}$; for example, the coquaternion $u=2+\mathbf{i}+\mathbf{k} \in \llbracket 2 \rrbracket$, but $P_{3}(u)=\mathbf{i}+\mathbf{k} \neq 0$. Hence, the zero $x=2$ (and any other zero in the set $\mathscr{Z}_{1}$ ) is neither an isolated zero nor an hyperboloidal zero of $P_{3}$.

Observe that, for any two elements $z=2+\alpha \mathbf{i}-\alpha \mathbf{k}$ and $z^{\prime}=2+\alpha^{\prime} \mathbf{i}-\alpha^{\prime} \mathbf{k}$ in $\mathscr{Z}_{1}$, their difference $z-z^{\prime}=\left(\alpha-\alpha^{\prime}\right) \mathbf{i}-\left(\alpha-\alpha^{\prime}\right) \mathbf{k}$ is singular, so there is no contradiction with Theorem 5 . One can also show that any element in the set

$$
\mathscr{Z}_{2}=\left\{\frac{3}{2}+\alpha \mathbf{i}+\frac{1}{2} \mathbf{j}+\alpha \mathbf{k}: \alpha \in \mathbb{R}\right\} \varsubsetneqq \llbracket \frac{3}{2}+\frac{1}{2} \mathbf{j} \rrbracket
$$

is a zero of $P_{3}$, but not all the elements in $\llbracket \frac{3}{2}+\frac{1}{2} \mathbf{j} \rrbracket$ are zeros of $P_{3}$. For example, the coquaternion $v=\frac{3}{2}+\mathbf{i}+\frac{1}{2} \mathbf{j}-\mathbf{k} \in \llbracket \frac{3}{2}+\frac{1}{2} \mathbf{j} \rrbracket$, but $P(v)=-\mathbf{i}+\mathbf{k} \neq 0$.

Apart from confirming our statement about the existence, in the case of coquaternionic polynomials, of zeros which are neither isolated nor hyperboloidal, this example also illustrates the statement made in 2.: we have a real zero, $x=2$, which is not an isolated zero of the coquaternionic polynomial $P_{2}$, a situation that cannot occur in the case of a quaternionic polynomial. 
Remark 10. We must also refer that this example contradicts the statement of [26, Theorem 2.4]: we have more than one zero in a class whose elements have a non-singular vector part — the class $\llbracket \frac{3}{2}+\frac{1}{2} \mathbf{j} \rrbracket-$ and, however, these zeros are not hyperboloidal.

\subsubsection{Acknowledgments}

Research at CMAT was financed by Portuguese Funds through FCT - Fundação para a Ciência e a Tecnologia, within the Project UID/MAT/00013/2013. Research at NIPE was carried out within the funding with COMPETE reference number POCI-01-0145-FEDER-006683 (UID/ECO/03182/2013), with the FCT/MEC's (Fundação para a Ciência e a Tecnologia, I.P.) financial support through national funding and by the ERDF through the Operational Programme on "Competitiveness and Internationalization - COMPETE 2020" under the PT2020 Partnership Agreement.

\section{References}

[1] Adler, S.: Quaternionic Quantum Mechanics and Quantum Fields, International Series of Monographs of Physics, vol. 88. Oxford University Press, New York (1995)

[2] Alagöz, Y., Oral, K.H., Yüce, S.: Split quaternion matrices. Miskolc Math. Notes 13, 223-232 (2012)

[3] Artzy, R.: Dynamics of quadratic functions in cycle planes. J. Geom. 44, 26-32 (1992)

[4] Brenner, J.L.: Matrices of quaternions. Pacific J. Math. 1(3), 329-335 (1951)

[5] Brody, D.C., Graefe, E.M.: On complexified mechanics and coquaternions. J. Phys. A: Math. Theory 44, 1-9 (2011)

[6] Cockle, J.: On systems of algebra involving more than one imaginary; and on equations of the fifth degree. Philos. Mag. 35(3), 434-435 (1849)

[7] De Leo, S., Ducati, G., Leonardi, V.: Zeros of unilateral quaternionic polynomials. Electron. J. Linear Algebra 15, 297-313 (2006)

[8] Falcão, M.I.: Newton method in the context of quaternion analysis. Appl. Math. Comput. 236, 458-470 (2014)

[9] Gordon, B., Motzkin, T.S.: On the zeros of polynomials over division rings. Trans. Am. Math. Soc. 116, 218-226 (1965)

[10] Gürlebeck, K., Sprößig, W.: Quaternionic Calculus for Engineers and Physicists. John Wiley\& Sons (1997)

[11] Hamilton, W.R.: A new species of imaginaries quantities connected with a theory of quaternions. Proc. R. Ir. Acad 2, 424-434 (1843)

[12] Janovská, D., Opfer, G.: Computing quaternionic roots in Newton's method. Electron. Trans. Numer. Anal. 26, 82-102 (2007)

[13] Janovská, D., Opfer, G.: The classification and the computation of the zeros of quaternionic, two-sided polynomials. Numer. Math. 115(1), 81-100 (2010)

[14] Janovská, D., Opfer, G.: Linear equations and the Kronecker product in coquaternions. Mitt. Math. Ges. Hamburg 33, 181-196 (2013)

[15] Janovská, D., Opfer, G.: Zeros and singular points for one-sided coquaternionic polynomials with an extension to other $\mathbb{R}^{4}$ algebras. Electron. Trans. Numer. Anal. 41, 133-158 (2014)

[16] Kalantari, B.: Algorithms for quaternion polynomial root-finding. J. Complex. 29, 302-322 (2013)

[17] Kula, L., Yayli, Y.: Split quaternions and rotations in semi euclidean space $E_{2}^{4}$. J. Korean Math. Soc. 44, 1313-1327 (2007) 
[18] Lam, T.Y.: A First Course in Noncommutative Rings. Springer (1991)

[19] Malonek, H.R.: Quaternions in applied sciences. A historical perspective of a mathematical concept. 17th Inter. Conf. on the Appl. of Computer Science and Mathematics on Architecture and Civil Engineering, Weimar (2003)

[20] Miranda, F., Falcão, M.I.: Modified quaternion Newton methods. Lecture Notes in Comput. Sci., 8579 (Part 1):146-161 (2014)

[21] Niven, I.: Equations in quaternions. Amer. Math. Monthly 48, 654-661 (1941)

[22] Özdemir, M.: The roots of a split quaternion. Appl. Math. Lett. 22(2), 258-263 (2009)

[23] Özdemir, M., Ergin, A.: Some geometric applications of split quaternions. In: Proc. 16th Int. Conf. Jangjeon Math. Soc. vol. 16, pp. 108-115 (2005)

[24] Özdemir, M., Ergin, A.: Rotations with unit timelike quaternions in Minkowski 3-space. J. Geometry Phys. 56(2), 322-336 (2006)

[25] Pogorui, A., Shapiro, M.: On the structure of the set of zeros of quaternionic polynomials. Complex Variables Theory Appl. 49(6), 379-389 (2004)

[26] Pogoruy, A.A., Rodríguez-Dagnino, R.: Some algebraic and analytical properties of coquaternion algebra. Adv. Appl. Clifford Algebras 20(1), 79-84 (2010)

[27] Serôdio, R., Siu, L.S.: Zeros of quaternion polynomials. Appl. Math. Letters 14(2), 237-239 (2001)

[28] Serôdio, R., Pereira, E., Vitória, J.: Computing the zeros of quaternion polynomials. Comput. Math. Appl. 42(8-9), 1229-1237 (2001)

[29] Shoemake, K.: Animating rotation with quaternion curves. SIGGRAPH Comput. Graph. 19(3), 245-254 (1985)

[30] Topuridze, N.: On roots of quaternion polynomials. J. Math. Sci. 160, 843-855 (2009) 